\title{
Efectos de la meteorización sobre los materiales de las vidrieras históricas
}

\section{Weathering effects on materials from historical stained glass windows}

Fecha de recepción: 2-XII-02

Fecha de aceptación: 8-I-03
M. GARCÍA-HERAS ${ }^{*}$, C. GIL**, N. CARMONA*, M. A. VILLEGAS "Centro Nacional de Investigaciones Metalúrgicas (CENIM), CSIC "Fundación Centro Nacional del Vidrio. Real Fábrica de Cristales

ESPAÑA

\section{RESUMEN}

Se ha caracterizado una selección de materiales (vidrios, perfiles de plomo, elementos de sujeción y masilla) procedentes de vidrieras históricas de distintos periodos (s. XIII - s. XIX). Las técnicas utilizadas fueron microscopía óptica, microscopía electrónica de barrido, espectrometría de rayos $X$ por dispersión de energías y difracción de ray'os $X$.

La degradación de las vidrieras históricas se debe a la composición química particular de los materiales que las constituyen: los vidrios coloreados, la red de emplomado, los elementos metálicos de sujeción y la masilla de relleno. La sola presencia de una determinada composición química no es, sin embargo, el único factor que interviene en el proceso de degradación. Es necesaria la concurrencia de causas externas que contribuyen a la propagación y. desarrollo masivo de las alteraciones de cada uno de los materiales que forman la vidriera. La presencia de gases contaminantes en la atmósfera provoca una interacción perjudicial con la superficie de los materiales de la vidriera. Los vidrios coloreados y grisallas comienzan por desalcalinizarse y destruir la capa de gel de sílice que se forma en un primer contacto con la humedad ambiental. Posteriormente se forman depósitos insolubles de sales y costras de corrosión, como consecuencia de un ataque químico más profundo que da lugar a la despolimerización de la red vítrea. La red de plomo y los elementos metálicos de sujeción también se deterioran por efecto de la meteorización, oxidándose y formando picaduras y costras de depósitos en su superficie. El arrastre de materiales procedentes de la costra de corrosión de los elementos metálicos da lugar a nuevos depósitos en los vidrios que pueden potenciar sus procesos de degradación. La masilla experimenta una contracción y agrietamiento considerables con el tiempo. Las condiciones ambientales adversas favorecen, asimismo, el transporte de sustancias de la masilla hacia los otros materiales de la vidriera, aumentando el espesor de sus costras y aportando elementos que se suman a la alteración global de la vidriera.

\begin{abstract}
SUMMARY
A selection of materials (stained glasses, lead cames, support elements and putty) from historical stained glass window's of different periods (13th-19th centuries) have been studied. Optical microscopy, scanning electron microscopy; energy dispersive X-ray spectrometry and X-ray diffraction were used as characterisation techniques.
\end{abstract}

PALABRAS CLAVE: metcorización, vidrieras históricas, vidrio, corrosión.
KEYWORDS: weathering, historic stained windows, glass, corrosion. 


\section{INTRODUCCIÓN}

De todos los aspectos del arte, las vidrieras constituyen uno de los más desconocidos a pesar de su capacidad de seducción y belleza. Asimismo, las vidrieras son un elemento arquitectónico que desempeña, cuando menos, una triple función: cerramiento de huecos, decoración de muros y filtro luminoso. A pesar de su funcionalidad y de que han sido elementos constantes en las edificaciones, con mayor o menor frecuencia, desde la Edad Media hasta nuestros días, las vidricras son fácilmente olvidadas o relegadas cuando se trata de programas de rehabilitación y restauración, que suelen omitir la importancia de su papel arquitectónico. Es posible que ello se deba a la generalizada consideración de que el vidrio, principal componente de las vidrieras, es un material químicamente estable y, salvo por su fragilidad mecánica, resistente al paso del tiempo. Sin embargo, el vidrio, o mejor, los vidrios de las vidrieras pueden experimentar, y de hecho han experimentado, diferentes procesos de degradación química que han ocasionado daños de diferente magnitud, a veces irreparables, dependiendo de las propias características del vidrio y de las condiciones ambientales de conservación.

Actualmente existen numerosos estudios sobre el proceso de degradación química de los vidrios procedentes de vidrieras históricas (1-6), y se puede afirmar que el mecanismo de ataque químico es conocido en sus distintas etapas (7). Por otro lado, el análisis de otros elementos y materiales que forman parte de las vidrieras, se ha llevado a cabo de un modo parcial y siempre más esporádicamente. Entre dichos materiales se pueden enumerar los siguientes: perfiles de plomo o emplomados, barras y trabillas metálicas de sujeción y masilla de relleno de las uniones entre emplomado y vidrio. Dichos materiales no constituyen elementos básicos artísticos ni decorativos de las vidrieras, pero son importantes desde el punto de vista práctico y técnico, pues son los que, en definitiva, dan cohesión y unidad estructural a la vidriera. Quizá sea interesante señalar que, en un número considerable de vidrieras, los perfiles de plomo han jugado un papel cstético en el conjunto, ya que los motivos iconográficos principales se solían resaltar con un perfil más ancho o con la superposición de dos perfiles de plomo que hacían el efecto de línea perfiladora, sobre todo cuando las vidrieras se contemplan desde el interior a contraluz.

El estado de conservación de los elementos metálicos y de la masilla, así como su resistencia química y física frente a la acción de la meteorización, de los contaminantes y de las agresiones de diversa índole, puede afectar directamente al grado de conservación de la vidriera en su conjunto o de los fragmentos de vidrios coloreados, en particular.

\section{INTRODUCTION}

Among all the art aspects, the stained glass windows are one of the most unknown, in spite of their beautifulness and seduction capability. Moreover, the stained glass windows are an architectural element that, at least, plays three functions: to close windows and walls; to decorate the walls; and to filter the light. Apart from these functionalities and taking into account that they were more or less usual elements in buildings from the Middle Age up to the present, stained glass windows are often forgotten or put away' in restoration programmes in which their architectural role is omitted. Maybe this is due to the general knowledge about glass, the main component of the stained glass windows, as a chemically stable material and, except for its mechanical fragility, a well resistant material against the pass of time. However, different chemical degradation processes could be and can be produced upon the stained glasses. Such degradation processes perform damages with different intensity; sometimes irreversible, depending on particular characteristics of the glass and environmental conservation conditions.

There are numerous papers about the chemical degradation process of stained glasses (1-6) and, on the other hand, the different steps of the chemical attack mechanism are well-known (7). In contrast, the analysis of other elements and materials from the stained glass windows has been scarcely performed. Among them, lead cames, metallic supporting bars and hooks, and refilling putty located in between the lead network and the stained glass pieces, can be considered. In the stained glass windows, these materials do not perform by themselves a basic artistic or decorative function. However, they are important as practical and technical materials since they give cohesion and structural identity to the stained glass window. It is also noticeable the fact that in many stained glass windows the lead came have played an aesthetic role in the assemble, since some main iconographic items were stick out by means of a wider lead came or by the union of two lead cames. The result was a larger line in between some of the stained glass pieces that improved the graphic impression, especially when the stained glass windows are observed against light from indoor.

The conservation state of metallic elements and putt;; as well as their chemical and physical resistance to weathering, pollutants and other aggressive circumstances, can affect directly the conservation degree of the whole stained glass window and, in particular, the conservation degree of the stained glass pieces. 
Puesto que el valor histórico y artístico de las vidrieras reside principalmente en los vidrios coloreados y decorados con grisallas, el resto de los elementos sustentantes ha sufrido un desprecio considerable, de modo que en las restauraciones se procede generalmente a sustituirlos cuando presentan problemas de integridad, huellas de corrosión avanzada o simplemente un aspecto poco agradable. A dichos elementos se les demanda su papel como sustentadores en detrimento de su interés como materiales históricos. Generalmente se considera que lo importante es que ningún fragmento de vidrio quede sin el apoyo del emplomado y que exista buen acoplamiento con la masilla de relleno. En cuanto a las barras y trabillas de sujeción, éstas deben sostenerse a la fachada y sujetar apropiadamente la vidriera en su conjunto para que resista la presión del viento y las agresiones mecánicas.

La meteorización de las vidrieras se extiende lógicamente a todos sus elcmentos y, además de la degradación que se produce en los fragmentos de vidrios coloreados, también se encuentran huellas de ataque químico en los emplomados, los elementos de sujeción y la masilla. La importancia del grado de deterioro de los elementos metálicos reside en el hecho de que pueda repercutir en la integridad de los fragmentos de vidrios coloreados, y en cuanto a la masilla, su degradación afecta indirectamente a los vidrios en el extremo de que aquélla se desprenda y deje a éstos sueltos dentro de la red del emplomado, con el riesgo de que puedan vibrar, moverse libremente o caerse.

En el presente trabajo el objetivo principal ha sido analizar la degradación por meteorización del conjunto de la vidriera y, por lo tanto, determinar su efecto concreto no sólo en los vidrios históricos coloreados, sino también en varios elementos metálicos (emplomados y elementos de sujeción) y masilla. Se pretende resaltar la necesidad de afrontar la conservación preventiva de las vidrieras históricas teniendo en cuenta el impacto ambiental que ya han experimentado y que puede agravarse seriamente con los actuales niveles de contaminación atmosférica. El objetivo final es poner de manifiesto la importancia de la vidriera histórica como un conjunto arquitectónico funcional, cuya conservación y restauración deben afrontarse con criterio integrador en cuanto a los elementos que la constituyen.

\section{EXPERIMENTAL}

Para la caracterización del deterioro químico y físico de vidrios, emplomados, barras y trabillas de sujeción y masilla, se han tomado diversas muestras históricas españolas de distinta procedencia, cuya cronología se extiende entre el s. XIII y el s. XIX.
Since the historical and artistic value of the stained glass windows is related to the stained glass pieces decorated or not with grisailles, all the other supporting elements have been scorned. In fact, in restoration tasks, they are usually substituted by other new ones when they show an advanced corrosion stage or a displeasing aspect. These materials are often considered as supporting elements rather than historic items. In this regard, it is generally accepted that the most important function of the lead network is to ensure the integrity of the stained glass pieces, in such a way: that a good junction between glasses and lead cames will be warranted. As far as the metallic bars and hooks are concerned, they have to be strongly adapted to the façade and support conveniently the whole stained glass window, with the aim that it can resist appropriately' wind pressure and mechanical impacts.

The stained glass windows weathering is obviously extended to all their components and, apart from the stained glass pieces degradation, weathering signs in the lead cames, supporting elements, and putty can be also found. The extent of the deterioration degree of the metallic elements determines indirectly the integrity of the stained glass pieces. In turn, the conservation state of the putty is important because their falling off would imply that the glass pieces would remain free inside the lead came, with the risk of vibrating and falling down.

In the present work the main objective has been to analyse the degradation by weathering of the whole stained glass window. Thus, we have study the weathering effect in the historical stained glass pieces, as well as in several metallic elements (lead cames and supporting elements) and putty: Likewise, our purpose is to point out the need of starting a preventive conservation of the stained glass windows, bearing in mind the environmental impact already suffered by them as well as the future conservation state that could be seriously affected under the present atmospheric pollution. The final objective is to show the relevance of the historical stained glass window as an architectural functional assemble, whose conservation and restoration have to be carried out with integrating criteria concerning all their components.

\section{EXPERIMENTAL}

In order to characterise the physical and chemical deterioration of stained glasses, lead cames, supporting bars and hooks, and putty, several Spanish historical samples from different provenances $\left(13^{\text {th }}-19^{\text {th }}\right.$ centuries) have been selected and studied. 
El análisis de la superficie de las muestras y de su sección transversal en algunos casos, se ha llevado a cabo mediante la observación por microscopía óptica (MO) con un microscopio Olympus modelo DP-11, y por microscopía electrónica de barrido (MEB) con un equipo JEOL modelo JXA-840 (15-20 kV), provisto de analizador de espectrometría de rayos X por dispersión de encrgías Rontec con detector de Si(Li). Las fases cristalinas presentes en las muestras y/o en sus costras de corrosión o depósitos superficiales se han detectado por difracción de rayos $\mathrm{X}(\mathrm{DRX})$ con un difractómetro Siemens modelo D-5000, con condiciones de trabajo de $40 \mathrm{kV}$ y $30 \mathrm{~mA}$. El análisis químico cuantitativo de la muestra de vidrio medieval se efectuó por espectrometría de emisión atómica mediante excitación de plasma acoplado inductivamente (ICP) y por fotometría de llama (FLL) según (7).

\section{RESULTADOS Y DISCUSIÓN}

Como es sabido (8), la alteración de la superficie de los vidrios y de las grisallas por el ataque químico de la humedad ambiental comienza con la formación de una capa de hidratación que da lugar a una película de gel de sílice relativamente porosa. Dicha película no es en principio perjudicial para la integridad del vidrio, sino que lo preserva de ulteriores agresiones. En la Figura 1 puede observarse una imagen de MEB de la superficic de un vidrio de procedencia arqueológica, donde aparece la capa de gel de sílice de aspecto escamoso. En dicha muestra se aprecia, asimismo, una zona en la que la capa de gel de sílice se ha desprendido dejando al descubierto la superficic del vidrio original, donde también se observan varias líneas de fractura de tipo concoideo. Cuando el medio ambiente se halla contaminado con gases de tipo ácido como el SO${ }_{2}, \mathrm{CO}_{2}$ y $\mathrm{NO}_{x}$, lo que es frecuente en los centros urbanos e industriales, se produce una combinación de la humedad con esos gases y se origina un medio rico en iones $\mathrm{H}_{3} \mathrm{O}^{+}$. A partir de ese momento, comienza un ataque ácido a la superficic del vidrio, en la que se produce un intercambio iónico entre los iones $\mathrm{H}^{+}$de los $\mathrm{H}_{3} \mathrm{O}^{+}$y los iones alcalinos del vidrio. La extracción de los iones alcalinos del vidrio o desalcalinización determina un aumento de la concentración de iones $\mathrm{OH}^{-}$ en el medio que hace aumentar $\mathrm{el} \mathrm{pH}$ desde valores ácidos hasta valores alcalinos. En la Tabla II se muestran los resultados de los microanálisis EDX realizados en las dos zonas representativas del vidrio de la Figura 1. Los contenidos de óxidos alcalinos y alcalinotérreos son en general menores en la capa de gel de sílice, lo que indica que se ha producido una desalcalinización en comparación con la composición química de la zona A donde se presenta la fractura concoidea y que puede corresponder al vidrio base. El

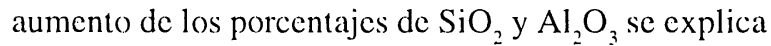
lógicamente por el enriquecimiento relativo que se
Superficial analyses of all the samples and, in some cases, transversal sections of them, have been performed by optical microscopy (OM) (Olympus microscope, mod. DP-II) and scanning electron microscopy (SEM) (JEOL unit mod. JXA -840, 15-20 $k V$, equipped with an energy dispersive $X$-ray spectrometer (EDX) Rontec with a Si(Li) detector). Crystalline phases in the samples and/or in the corrosion crusts or superficial deposits have been detected by X-ray diffraction (XRD) (Siemens diffractometer mod. D-5000, $40 \mathrm{kV}$ and $30 \mathrm{~mA}$ ). The quantitative chemical analysis of the medieval glass sample was done by inductive coupled plasma (ICP) and flame photometry (FP), after (7).

\section{RESULTS AND DISCUSSION}

As is known (8), the superficial alteration of glasses and grisailles by the chemical attack performed by environmental humidity begins with the formation of a hidratation layer that forms a relatively porous silica gel film. In a first approach, that silica gel film preserves the glass against further chemical damage rather than affects the glass integrity: In Figure 1, a SEM image from a glass surface (archaeological provenance) can be observed. The silica gel layer appears delaminated, to some extent, in the upper zone. The surface of the original glass is observed at the bottom of Figure 1, where some concave fracture lines appear. If the environment contains acidic gaseous pollutants such as $\mathrm{SO}_{2}, \mathrm{CO}_{2}$ and $\mathrm{NO}_{r}$, which frequently occurs in urban and industrial centres, the combination of humidity and gaseous pollutants gives rise to a $\mathrm{H}_{3} \mathrm{O}^{+}$-ions rich medium. As a consequence, an acid attack starts in the glass by the ion exchange of $\mathrm{H}^{+}$ from $\mathrm{H}_{3} \mathrm{O}^{+}$with the alkaline ions from the glass. The alkaline ions leaching or dealkalinisation determines an increase of $\mathrm{OH}$-ions in the surrounding medium, increasing the $\mathrm{pH}$ from acid to alkaline values. Table II offers the EDX microanalyses results derived from two representative zones of the glass shown in Figure 1. On the one hand, the alkaline and earth-alkaline oxides contents decreased in the silica gel layer, which indicates that a dealkalinisation process took place. On the other hand, percentages of oxides detected in zone A of Figure 1 correspond to the chemical composition of the original glass. The increase of $\mathrm{SiO}_{2}$ and $\mathrm{Al}_{2} \mathrm{O}_{3}$ percentages can be explained by the relative 
produce de dichos óxidos al disminuir los contenidos de óxidos alcalinos y alcalinotérreos.

Cuando el medio en el que se encuentra el vidrio no se renueva, cs decir, si se manticne en condiciones estáticas en cuanto a humedad relativa clevada y presencia de gases contaminantes, cl ataque químico prosigue en medio alcalino. En este caso los iones $\mathrm{OH}^{-}$ rompen las uniones siloxano $(\mathrm{Si}-\mathrm{O}-\mathrm{Si})$ de la red vítrea dando lugar a un proceso de despolimerización y destrucción del vidrio. Simultáneamente se forman depósitos de sales insolubles, sobre todo de sulfatos y carbonatos, que se forman por combinación de los iones alcalinos y alcalinotérreos extraídos con los gases contaminantes $\mathrm{SO}_{2}$ y $\mathrm{CO}_{2}$, respectivamente. En la Figura 2 se ilustra el aspecto de un vidrio procedente de una vidricra medieval (s. XIII). La superficie aparece con picaduras y depósitos, además de una gruesa capa de suciedad y productos de la degradación química. Los depósitos insolubles más frecuentes en los vidrios históricos son los siguientes: yeso $\mathrm{CaSO}_{4} \cdot 2 \mathrm{H}_{2} \mathrm{O}$, bassanita $\mathrm{CaSO}_{4} \cdot 1 / 2 \mathrm{H}_{2} \mathrm{O}$, singenita $\mathrm{K}_{2} \mathrm{Ca}\left(\mathrm{SO}_{4}\right)_{2} \cdot \mathrm{H}_{2} \mathrm{O}$ y calcita $\mathrm{CaCO}_{3}$. En el caso del vidrio de la Figura 2, se comprobó por EDX (7) que los depósitos pueden corresponder a carbonato cálcico formado tras una desalcalinización intensa de los iones $\mathrm{K}^{+}$y $\mathrm{Ca}^{2+} \mathrm{del}$ vidrio. En la Tabla I se muestran los resultados del análisis químico cuantitativo de dicho vidrio realizado por ICP. Dichos resultados se comparan con los obtenidos mediante EDX. Pucde comprobarse que se trata de un vidrio potásico-cálcico, que se ajusta a la composición tipo de los vidrios medievales, con elevados porcentajes de $\mathrm{K}_{2} \mathrm{O}$ y $\mathrm{CaO}$, y un contenido de $\mathrm{SiO}_{2}$ relativamente bajo $(50,9 \%$ en peso). Por lo tanto es un vidrio con una estabilidad química baja. En los vidrios medievales es frecuente un enriquecimiento superficial de $\mathrm{SiO}_{2}$, como consecuencia de la correspondiente disminución de los porcentajes de óxidos alcalinos y alcalinotérreos, debida al proceso de desalcalinización descrito.

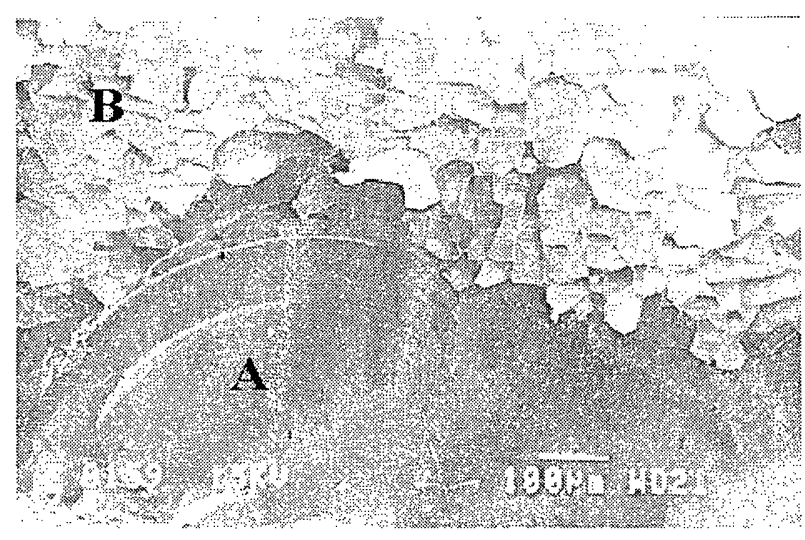

Figura 1.- Imagen de MEB de la superficie de un vidrio de procedencia arqueológica.

Figure 1.- SEM image of the surface of an archaeological glass. enrichment of such oxides, due to the alkaline and earth-alkaline oxides decreasing.

When the glass surrounding remains unchanged (static conditions), with high relative humidity and gaseous pollutants, the chemical attack continues, following a mechanism of glass corrosion under alkaline medium. In this case, the $O H_{-i o n s}$ break down the siloxane bonds (Si-O-Si) of the glass network, which signifies the depolymerisation of this network. Simultaneously; insoluble salt deposits appear, mainly sulphates and carbonates, formed by combination of leached alkaline and earth-alkaline ions with gaseous pollutants, e.g. $\mathrm{SO}_{2}$ and $\mathrm{CO}_{2}$, respectively: Figure 2 depicts the aspect of a glass from a medieval stained glass window (1 $3^{\text {th }}$ century). The surface shows pits and deposits and a thick crust due to dirty and accumulation of chemical degradation products. The most frequent insoluble deposits on historical glasses are the following: gypsum $\mathrm{CaSO}_{4} \cdot 2 \mathrm{H}_{2} \mathrm{O}$, bassanite $\mathrm{CaSO}_{4} \mathrm{I}_{2} \mathrm{H}_{2} \mathrm{O}$, syngenite $\mathrm{K}_{2} \mathrm{Ca}\left(\mathrm{SO}_{4}\right)_{2} \cdot \mathrm{H}_{2} \mathrm{O}$ and calcite $\mathrm{CaCO}_{3}$. As was confirmed by $\operatorname{EDX}(7)$, deposits of the glass shown in Figure 2 can be attributed to calcium carbonate formed after a strong dealkalinisation of $\mathrm{K}^{+}$and $\mathrm{Ca}^{2+}$-ions from the glass. In Table I, the results of quantitative chemical analysis performed by ICP on such a glass are shown. These results are also compared with those obtained by EDX on the same sample. As Table I indicates, it is a potassium-lime silicate glass, whose composition fits well with the medieval type-like glasses: high percentage of $\mathrm{K}_{2} \mathrm{O}$ and $\mathrm{CaO}$, and $\mathrm{a}$ relatively low $\mathrm{SiO}_{2}$ content $(50.9 \mathrm{wt}$. \%). Consequently, this is a low chemical stability glass. In medieval glasses some superficial $\mathrm{SiO}_{2}$ enrichment is frequent, due to the alkaline and earth-alkaline oxides diminishing during the dealkalinisation process described.

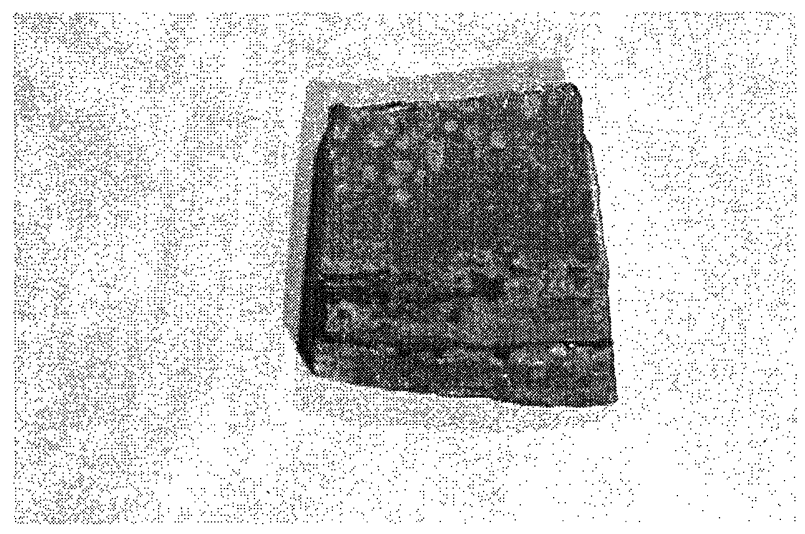

Figura 2.- Fotografía de la superficie de un vidrio procedente de una vidriera medieval (s. XIII).

Figure 2.- Photograph of a glass surface from a medieval stained glass window (13 th (entury). 
TABLA I/TABLE I

Resultados de los análisis químicos del vidrio de la Figura 2, realizados mediante ICP, FLL y EDX

Chemical analyses results of glass shown in Figure 2, obtained by ICP, Fp and EDX

\begin{tabular}{|c|c|c|c|c|c|c|c|c|c|c|c|c|}
\hline $\begin{array}{c}\text { Óxidos } \\
\text { (\%peso) } \\
\text { Oxides } \\
\text { (wt. \%) }\end{array}$ & $\mathrm{P}_{2} \mathrm{O}_{5}$ & $\mathrm{SiO}_{2}$ & $\mathrm{Al}_{2} \mathrm{O}_{3}$ & $\mathrm{Fe}_{2} \mathrm{O}_{3}$ & $\mathrm{MgO}$ & $\mathrm{CaO}$ & $\mathrm{MnO}$ & $\mathrm{Na}_{2} \mathrm{O}$ & $\mathrm{K}_{2} \mathrm{O}$ & $\mathrm{SO}_{3}$ & $\mathrm{Cl}^{-}$ & $\begin{array}{c}\text { Otros } \\
\text { Others }\end{array}$ \\
\hline ICP & 3,7 & 50,9 & 0,6 & 0,4 & 6,8 & 14,1 & 1,6 & 3,2 & 17,2 & 0,3 & - & 1,2 \\
\hline EDX & 4,9 & 49,7 & 1,4 & 1,0 & 4,1 & 15,6 & 2,5 & 0,9 & 18,9 & 0,7 & 0,3 & - \\
\hline
\end{tabular}

TABLA II/TABLE II

Resultados de los microanálisis EDX realizados en las distintas muestras estudiadas. Los resultados correspondientes a azufre y plomo se han expresado conjuntamente, cuando coexisten ambos, debido al solapamiento que presentan dichos elementos en los espectros de EDX EDX microanlyses results perfomed on the samples studied. Results for sulphur and lead have been put together since they coexist, due to the overlapping of such elements in the EDX spectra

\begin{tabular}{|c|c|c|c|c|c|c|c|c|c|c|}
\hline \multirow{2}{*}{$\begin{array}{c}\text { Muestras } \\
\text { Samples }\end{array}$} & \multicolumn{7}{|c|}{ Óxidos (\% peso) Oxides (wt. \%) } \\
\cline { 2 - 13 } & $\mathrm{SiO}_{2}$ & $\mathrm{Al}_{2} \mathrm{O}_{3}$ & $\mathrm{CaO}$ & $\mathrm{MgO}$ & $\mathrm{Na}_{2} \mathrm{O}$ & $\mathrm{K}_{2} \mathrm{O}$ & $\mathrm{Fe}_{2} \mathrm{O}_{3}$ & $\mathrm{SO}_{2}$ & $\mathrm{PbO}$ \\
\hline $\begin{array}{l}\text { Fig. 1 zona A } \\
\text { Fig. 1 zone A }\end{array}$ & 59,1 & 9,7 & 9,8 & 5,2 & 11,4 & 3,3 & 1,5 & - & - \\
\hline $\begin{array}{l}\text { Fig. 1 zona B } \\
\text { Fig. 1 zone B }\end{array}$ & 64,5 & 18,8 & 5,4 & 3,4 & 2,8 & 4,5 & 0,6 & - & - \\
\hline $\begin{array}{l}\text { Fig. 3 zona A } \\
\text { Fig. 3 zone A }\end{array}$ & 26,2 & 5,9 & 55,3 & 1,5 & 0,5 & 2,4 & 2,5 & \multicolumn{3}{|c|}{5,7} \\
\hline $\begin{array}{l}\text { Fig. 3 zona B } \\
\text { Fig. 3 zone B }\end{array}$ & 66,0 & 2,1 & 18,8 & 0,6 & 8,1 & 0,9 & 0,7 & \multicolumn{3}{|c|}{2,8} \\
\hline $\begin{array}{l}\text { Fig. 12 zona A } \\
\text { Fig. 12 zone A }\end{array}$ & 3,2 & 0,4 & 9,9 & - & - & - & - & - & 86,5 \\
\hline $\begin{array}{c}\text { Muestras } \\
\text { Samples }\end{array}$ & $\mathrm{Si}$ & $\mathrm{Al}$ & $\mathrm{Ca}$ & $\mathrm{K}$ & $\mathrm{Fe}$ & $\mathrm{S}$ & $\mathrm{Cl}$ & $\mathrm{P}$ & $\mathrm{Pb}$ & $\mathrm{O}$ \\
\hline $\begin{array}{l}\text { Fig. 8 zona A } \\
\text { Fig. 8 zone A }\end{array}$ & 14,8 & 8,0 & 14,3 & 1,1 & 2,1 & - & - & 3,1 & 3,8 & 52,8 \\
\hline $\begin{array}{l}\text { Fig. 8 zona B } \\
\text { Fig. 8 zone B }\end{array}$ & 2,8 & 0,8 & 1,5 & - & - & - & - & 10,2 & 24,4 & 60,3 \\
\hline $\begin{array}{l}\text { Fig. 8 zona C } \\
\text { Fig. 8 zone C }\end{array}$ & - & - & - & - & - & - & - & - & 65,2 & 34,8 \\
\hline $\begin{array}{l}\text { Fig. 10 zona A } \\
\text { Fig. 10 zone A }\end{array}$ & 0,8 & - & - & - & 98,0 & 1,2 & - & - & - & - \\
\hline $\begin{array}{l}\text { Fig. 10 zona B } \\
\text { Fig. 10 zone B }\end{array}$ & 0,3 & - & 0,3 & - & 43,6 & - & 2,2 & - & - & 53,6 \\
\hline
\end{tabular}


La Figura 3 muestra una imagen de MEB correspondiente a un vidrio procedente de una vidriera del s. XIX en el que se presenta una costra de aspecto terroso. El microanálisis de EDX (Tabla II) realizado en la zona A de la Figura 3, puede indicar que, a pesar de tratarse de un vidrio sódico cálcico de mayor estabilidad química que los medievales potásico-cálcicos, no puede descartarse una desalcalinización, como indicarían los bajos contenidos de óxidos alcalinos. Por otro lado, el elevado porcentaje de óxido de calcio y la presencia de azufre $\left(\mathrm{SO}_{2}\right)$ pueden deberse a la formación de una costra de sulfato de calcio o a depósitos de restos de mortero. En la zona B (Figura 3) el microanálisis EDX (Tabla II) indicó, asimismo, una elevada proporción de óxido de calcio, la presencia de azufre y mayores porcentajes de sílice, que pueden interpretarse de un modo similar a la situación de la zona $\mathrm{A}$, aunque en un avance menor del proceso de degradación química.

En cuanto a las grisallas (decoraciones de pinturas vitrificables), éstas pueden experimentar un proceso de alteración y degradación química semejante al de los vidrios base, puesto que están formadas por óxidos de plomo, silicio, calcio y hierro (Tabla III), que pueden ser asimismo componentes habituales en los vidrios. Sin embargo, su estado de conservación es muy variable dependiendo de su composición concreta, de su posición en la cara interna o en la externa de la vidricra, de la intensidad de la metcorización sufrida, etc. En la Figura 4 se presenta una imagen frontal de MEB de la grisalla de un vidrio procedente de una vidricra del s. XIX. Se observa una textura relativamente homogénea de aspecto poroso que corresponde al residuo vitrificado de la pintura, preparada probablemente a partir de una suspensión de sales metálicas o de

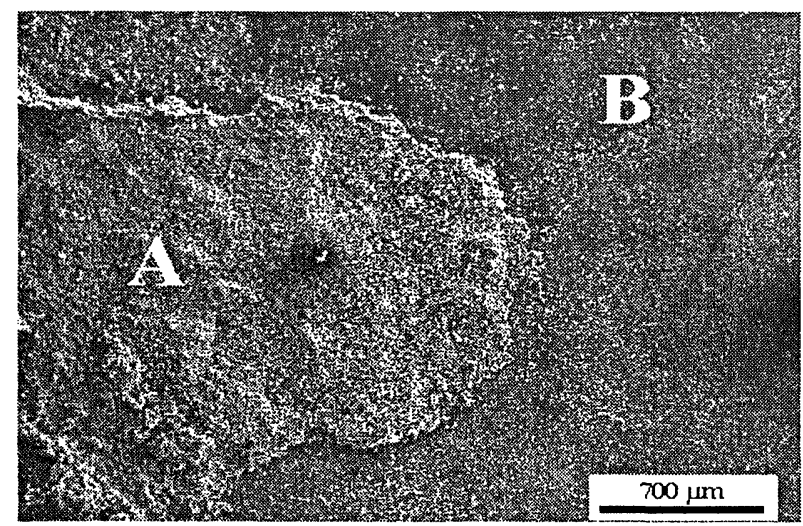

Figura 3.- Imagen de MEB de la superficie de un vidrio procedente de una vidriera del s. XIX.

Figure 3.- SEM image of a glass surface from a $19^{\text {th }}$ century stained glass window.
Figure 3 shows a SEM image of a glass piece from a stained glass window of $19^{\text {th }}$ century: As EDX microanalysis on zone A indicates, some dealkalinisation degree can not be discarded (see the low alkaline oxides content), in spite that its composition is of a soda lime silicate glass, stabler than those of medieval potassium-lime silicate glasses. In addition, the high percentage of calcium oxide and the presence of $\mathrm{SO}_{2}$ could be due to the crust formed by $\mathrm{CaSO}_{4}$ or by deposits from residual mortar. In zone $\mathrm{B}$ of Figure 3, EDX microanalyses (Table II) show a high amount of $\mathrm{CaO}$, the presence of sulphur, and higher percentages of $\mathrm{SiO}_{2}$. Such results could be addressed in a similar way as in zone $A$, even though a minor advance in the chemical degradation process is oberved.

The grisailles (decoration made with glassy paints) can submit to a chemical alteration and degradation similar to that in base glasses, since they are constituted by lead, silicon, calcium and iron oxides (Table III), as some glasses do. However, their conservation state is variable depending on their particular composition, their position in the stained glass window (indoor or outdoor), the weathering intensity; etc. In Figure 4 a front SEM image of a $19^{\text {th }}$ century stained glass window grisaille is shown. A relatively homogeneous and porous texture corresponding to the glassy residue of the paint can be observed. The grisaille was probably prepared from a suspension of metallic salts or minerals together with

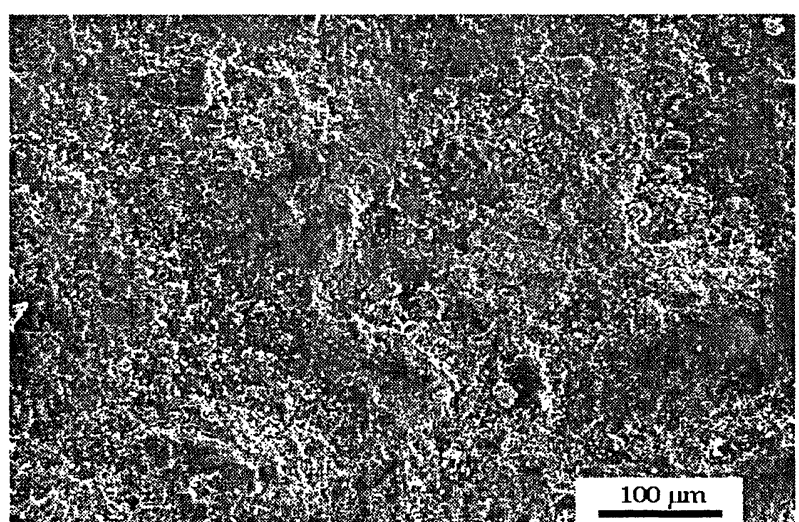

Figura 4.- Imagen de MEB de la grisalla (gris oscuro) de un vidrio procedente de una vidricra del s. XIX.

Figure 4.- SEM image of the grisaille (dark grey') of a glass from a $19^{\text {th }}$ century stained glass window: 
TABLA III/TABLE III

Composición química promedio de grisallas procedentes de vidricras medievales (7).

Resultados obtenidos mediante EDx

Average chemical composition of grisailles from medieval stained glass windows (7).

Results were obtained by EDX

\begin{tabular}{|l|c|c|c|c|c|c|c|c|c|}
\hline $\begin{array}{l}\text { Óxidos } \\
\text { Oxides }\end{array}$ & $\mathrm{PbO}$ & $\mathrm{Fe}_{2} \mathrm{O}_{3}$ & $\mathrm{SiO}_{2}$ & $\mathrm{CaO}$ & $\mathrm{P}_{2} \mathrm{O}_{5}$ & $\mathrm{Al}_{2} \mathrm{O}_{3}$ & $\mathrm{SO}_{2}$ & $\mathrm{~K}_{2} \mathrm{O}$ & $\mathrm{CuO}$ \\
\hline $\begin{array}{l}\text { \% peso } \\
\text { (wt. \%) }\end{array}$ & 23,6 & 21,0 & 20,7 & 17,1 & 4,9 & 4,6 & 3,5 & 2,6 & 2,0 \\
\hline
\end{tabular}

minerales en un vehículo orgánico como aceite de linaza. La descomposición térmica de los componentes orgánicos y su volatilización dan lugar a la microestructura porosa que se observa en la Figura 4. Es posible que las grisallas, en especial las del período medieval, igual que los vidrios coloreados experimenten un proceso de deterioro químico, como la extracción de algunos de sus clementos alcalinos o alcalinotérreos que podrían formar depósitos insolubles $\left(\mathrm{CaCO}_{3}, \mathrm{CaSO}_{4}\right)$. Otras veces es frecuente que las grisallas se desprendan del vidrio como consecuencia de la penetración y avance de la corrosión en la intercara vidrio-grisalla. Asimismo, es posible encontrar grisallas de más de siete u ocho siglos de antigüedad que se han conservado mucho mejor que los correspondientes vidrios sobre los que se encuentran aplicadas. Este hecho se pucde explicar por la mayor estabilidad química respecto a la de los vidrios base que, en general, son vidrios medievales potásico-cálcicos y, por lo tanto, poco resistentes a la meteorización (8).

Los dos factores más importantes que determinan la gravedad del ataque químico que pueden experimentar los vidrios de las vidrieras históricas por efecto de la metcorización son su composición química y la agresividad del medio ambiente (humedad relativa y presencia de gases contaminantes). En efecto, los vidrios en cuya composición hay más de un $60 \%$ en peso de $\mathrm{SiO}_{2}$, principal formador de la red vítrea, son estables frente a la meteorización (9) y en su superficie se puede observar la acumulación de suciedad, polvo y hollín que pueden limpiarse cuidadosamente sin mayores problemas, preservando la capa protectora de gel de sílice. En dichos vidrios no se observa la formación de costras de corrosión ni la aparición de picaduras o cráteres. Los vidrios de las vidricras del siglo XII, de las vidrieras renacentistas y de las vidrieras modernas de los siglos XIX y XX pertenecen a este tipo de vidrios (Figuras 5 y 6). Sin embargo, los vidrios de los siglos XIII y XIV poscían una proporciones de $\mathrm{SiO}_{2}$ y de otros formadores de la red vítrea (como $\mathrm{Al}_{2} \mathrm{O}_{3}$ ) menores del $60 \%$ y esto determinó un profundo deterioro y la aparición de gruesas costras formadas por productos insolubles de la corrosión química producida (Figura 2). an organic solvent as linseed oil. Thermal decomposition of organic components and further volatilisation yield the porous microstructure observed in Figure 4. It is possible that grisailles, especially. those from medieval times, submit to a chemical deterioration process similar to that of coloured glasses: for instance, the leaching of some of their alkaline or earth-alkaline elements, which could form insoluble deposits $\left(\mathrm{CaCO}_{3}, \mathrm{CaSO}_{4}\right)$. It is also frequent that grisailles were fallen off from the glass piece, due to the penetration and progress of the corrosion at the interface glass-grisaille. Likewise, one can find grisailles from seven or eight centuries ago with better conservation state than that of the glasses in which they were deposited. This fact can be explained by their higher chemical stability compared with those of the base glasses (medieval potassium-lime silicate glasses very sensitive against weathering) (8).

Two factors can be considered as the most important in determining the intensity of the chemical attack submitted by the stained window glasses as a result of weathering. They are the glass chemical composition and the environmental aggressiveness (relative humidity and gaseous pollutants). Thus, the glasses containing more than $60 \mathrm{wt}$. \% of $\mathrm{SiO}_{2}$, the main glass network forming, are stable against weathering (9). In their surface one can find dirty; dust and soot that can be carefully removed preserving the protective silica gel layer without additional problems. In these glasses, there are not corrosion crusts, pits or craters. Stained glasses from $12^{\text {th }}$ century; from Renaissance and modern stained glass windows, can be included in that type of glasses (Figures 5 and 6). However, the $13^{\text {th }}$ and $14^{\text {th }}$ centuries glasses had a content in glass network forming oxides $\left(\mathrm{SiO}_{2}, \mathrm{Al}_{2} \mathrm{O}_{3}\right)$ lower than $60 \mathrm{wt}$. \%, which determined a strong deterioration and the formation of thick crusts formed by insoluble products from the chemical corrosion (Figure 2). 


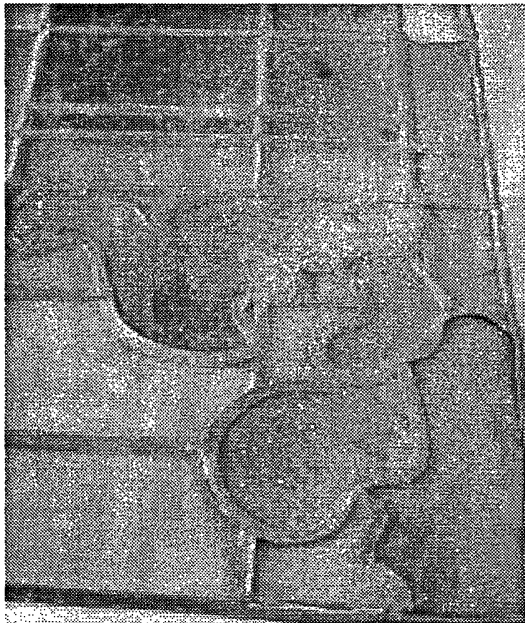

Figura 5.- Fotografía de un fragmento de vidriera del s. XIX cuyos vidrios coloreados aparecen cubiertos con una gruesa capa de suciedad.

Figure 5.- Photograph of a piece of stained glass window $\left(19^{\text {th }}\right.$ century) whose coloured glasses appear coated by a thick dirty layer:

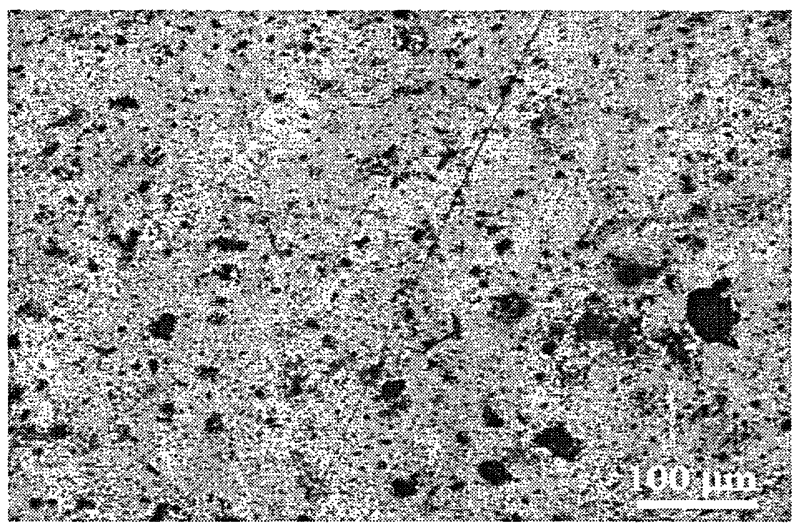

Figura 6.- Imagen de MO de la superficie de un vidrio procedente de una vidriera del s. XIX que presenta partículas adheridas de hollín.

Figure 6.- OM image of a glass surface from a $19^{\text {th }}$ century stained glass windou; showing soot particles adhered.
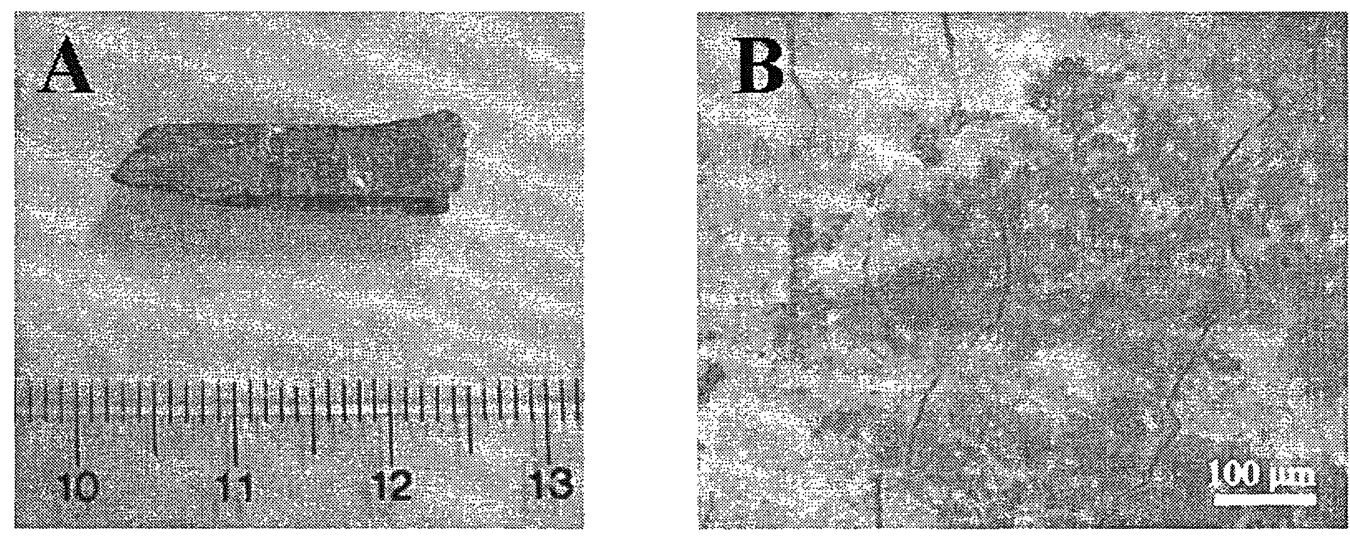

Figura 7.- A) Fotografía de un fragmento de emplomado procedente de una vidriera medieval. B) Imagen de MO del mismo fragmento de cmplomado.

Figure 7.- A) Photograph of a lead came piece from a medieval stained glass window: B) OM image of the same lead came piece.

A diferencia del volumen de investigaciones dedicadas a los vidrios de las vidrieras, los emplomados han recibido una atención escasa por parte de los especialistas. Algunos trabajos anteriores $(10,11,12)$ pusieron de manifiesto el interés de los perfiles de emplomado como material histórico constituyente de las vidrieras artísticas y su papel no sólo como elemento sustentador, sino también como potenciador del efecto estético de la iconografía representada y como participante en el proceso global de deterioro de la vidriera.

En la Figura 7A se muestra una fotografía de un fragmento de emplomado procedente de una vidriera medieval (s. XIV) donde puede apreciarse un grado de alteración superficial considerable y la formación de una costra. En la Figura 7B la imagen de MO de dicho fragmento presenta algunos detalles de la textura superficial que, además de los evidentes depósitos y
In contrast with the research undertaken about stained glasses, the lead cames have been scarcely investigated by specialists. Previous works $(10,11,12)$ have pointed out the interest of lead cames as a historical material in artistic stained glass windows. They have also claimed for the role of lead cames as an aesthetic element in the whole iconographic motives, as well as a material participating in the general deterioration process of the stained glass window.

In Figure 7A, a photograph of a lead came piece from a medieval stained glass window (14 th century) is shown. A great alteration degree and corrosion crust can be observed at the surface. In Figure 7B, an OM 
capa de oxidación generalizada, pone de manifiesto la existencia de microgrietas intcrconectadas. El origen de dichas grictas puede ser el choque térmico al que están sometidas las vidrieras en los sucesivos ciclos díanoche y verano-invierno, que induce dilataciones y contracciones de la red de emplomado. Las grietas son el resultado de las tensiones mecánicas producidas por dichos ciclos, ya que los coeficientes de dilatación del plomo y de los vidrios son distintos. A su vez la existencia de grictas favorece un posterior ataque químico por la humedad y los agentes contaminantes del ambiente.

Por otro lado, las observaciones llevadas a cabo mediante MEB en una sección transversal del mismo fragmento (Figura 8) demuestran que la capa adyacente al interior del perfil de plomo estudiado (zona B, aproximadamente $10 \mu \mathrm{m}$ de espesor) ticne un aspecto esponjoso comparado con la parte interna (zona C) y que corresponde fundamentalmente a $\mathrm{PbO}$, como se puede deducir del correspondiente microanálisis EDX (Tabla II). En la parte más externa de la sección transversal (zona A, aproximadamente $30 \mu \mathrm{m}$ de espesor), se observa una capa más oscura de aspecto relativamente compacto, cuyo microanálisis EDX indicó la presencia de varios elementos que hacen disminuir relativamente el porcentaje de plomo. En cuanto a las fases cristalinas de la costra (zona A), se pudo detectar por DRX que éstas corresponden a anglesita $\left(\mathrm{PbSO}_{4}\right)$ y, en menor proporción, a óxido de plomo litharge $(\mathrm{PbO})$ y a lanarkita $\left(\mathrm{Pb}_{2} \mathrm{OSO}_{4}\right)$, además de plomo metálico $(\mathrm{Pb})$, probablemente arrastrado durante el raspado de la muestra para aislar la costra externa. Todas las fases cristalinas detectadas presentaron un grado de cristalinidad muy bajo como es de esperar en este tipo de depósitos policristalinos formados por meteorización espontánca. Los contenidos relativamente altos de calcio y de silicio que se detectan por EDX en la zona A (Tabla II) podrían deberse a restos de masilla o del mortero; igualmente, la presencia de hierro se podría explicar por el arrastre de aquellos iones desde los elementos de sujeción.

Las barras y trabillas metálicas de sujeción han sido tan escasamente estudiadas como los perfiles de plomo. La interacción de dichos elementos metálicos con los vidrios coloreados es, sin embargo, importante, ya que aparte de su función de refuerzo mecánico del conjunto de la vidriera, ejercen un efecto sinérgico en el proceso de deterioro quimicofísico de los vidrios.

Frecuentemente se ha detectado sobre la superficie de los vidrios de vidrieras históricas fases cristalinas o elementos que sin duda provienen de la corrosión por meteorización de los elementos sustentantes, en especial del hicrro de las barras de sujeción. La lixiviación de iones metálicos, su arrastre por el agua de image depicts some superficial texture details: apart from deposits and general oxidation layer, interconnected microcracks can be observed. Their origin could be related to the thermal shock produced by successive day-night and summer-winter cycles, which induce dilatation and shrinkage in the lead came. Cracks result as a consequence of the mechanical stresses by such cycles, since linear expansion coefficients of lead and glasses are different. In turns, the presence of cracks favours further chemical attack performed by humidity and environmental pollutants.

\section{Furthermore, SEM observations carried out on a} section of the same lead piece (Figure 8), demonstrate that the layer near the inner zone of the came (zone $B$, at about $10 \mu \mathrm{m}$ thickness) has a sponge-like microstructure when compared with the inner zone $C$ (mainly formed by $\mathrm{PbO}$, as the EDX microanalysis showed, Table II). Upon the outer zone A (at about $30 \mu m$ thickness), a dark and compact layer can be observed. Its EDX microanalysis showed the presence of several elements that relatively diminish the lead percentage. In regarding the crystalline phases of the crust (zone $\mathrm{A})$, anglesite $\left(\mathrm{PbSO}_{4}\right)$ and low content of lead oxide litharge $(\mathrm{PbO})$, lanarkite $\left(\mathrm{Pb}_{2} \mathrm{OSO}_{4}\right)$ and metallic lead $(\mathrm{Pb})$ (probably pulled out during the scraping of the crust) were detected by XRD. All the crystalline phases detected showed a low crystallinity degree, as is usual in such a type of polycrystalline deposits formed by spontaneous weathering. The relatively high contents of calcium and silicon detected by EDX in zone A (Table II) could be due to residual putty or mortar. On the contrary, the iron presence could be explained by their dragging from supporting elements.

Supporting metallic bars and hooks, as the lead cames, have been also rarely studied. However, the interaction of such metallic elements with coloured glasses is important due to two reasons: their mechanical reinforcing role in the whole stained glass window and the synergic effect that they can do in the chemical and physical deterioration process of the glasses.

Frequently; crystalline phases or ions coming from the weathering corrosion products of the supporting elements (i.e. iron from the supporting bars) have been detected on the surface of the stained glasses. Metallic ions leaching, their dragging out by rain water and their deposition upon the coloured glasses, favours in 
lluvia y su depósito sobre los vidrios coloreados contribuye de forma significativa a incrementar el espesor de las costras de corrosión de los vidrios y, probablemente, dichos iones metálicos participan en los procesos de recristalización que se producen en ellas, combinándose con otros elementos y compuestos.

En la Figura 9A se muestra el aspecto de un fragmento de barra de sujeción de hierro procedente de una vidriera prerrenacentista (segunda mitad del s. XV). Se puede observar la existencia de una capa de corrosión extendida por toda la superficie con depósitos abundantes y aspecto muy heterogéneo. Las observaciones de MO de un fragmento de trabilla de sujeción (Figura 9B) demuestran efectivamente la presencia de diferentes texturas y grados de porosidad, lo que indica la concurrencia de varios compuestos some great extent corrosion crusts thickness in the glass pieces. Probably; those metallic ions play a role in the recrystallization processes that take place in the glasses by combination with other elements and compounds.

In Figure 9A, the appearance of a supporting iron bar piece from a pre-Renaissance stained glass window (15 th century) is shown. The presence of a corrosion layer extended by the entire surface with many deposits of heterogeneous aspect can be observed. The OM observations of a supporting hook piece (Figure 9B) demonstrate as well the presence of different textural areas with a different porosity degree. This indicates the formation of several compounds, as a result of

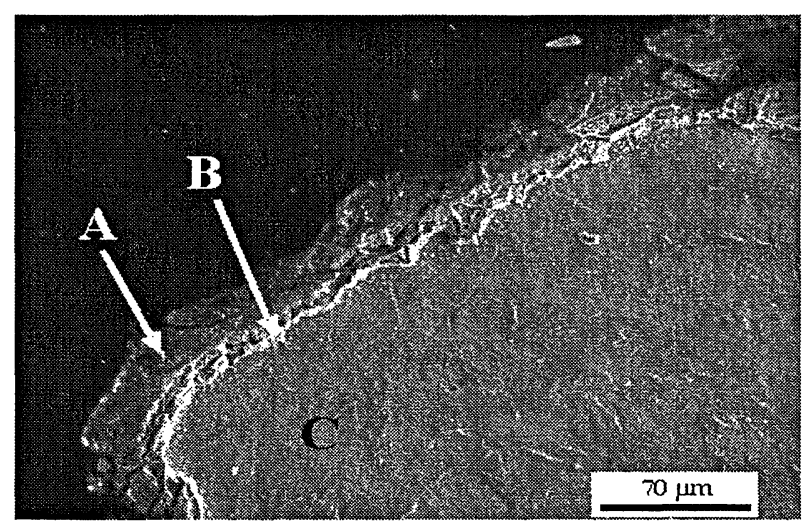

Figura 8.- Imagen de MEB de una sección transversal del fragmento de emplomado de la Figura 7.

Figure 8.- SEM image of a transversal section of the lead came piece of Figure 7.
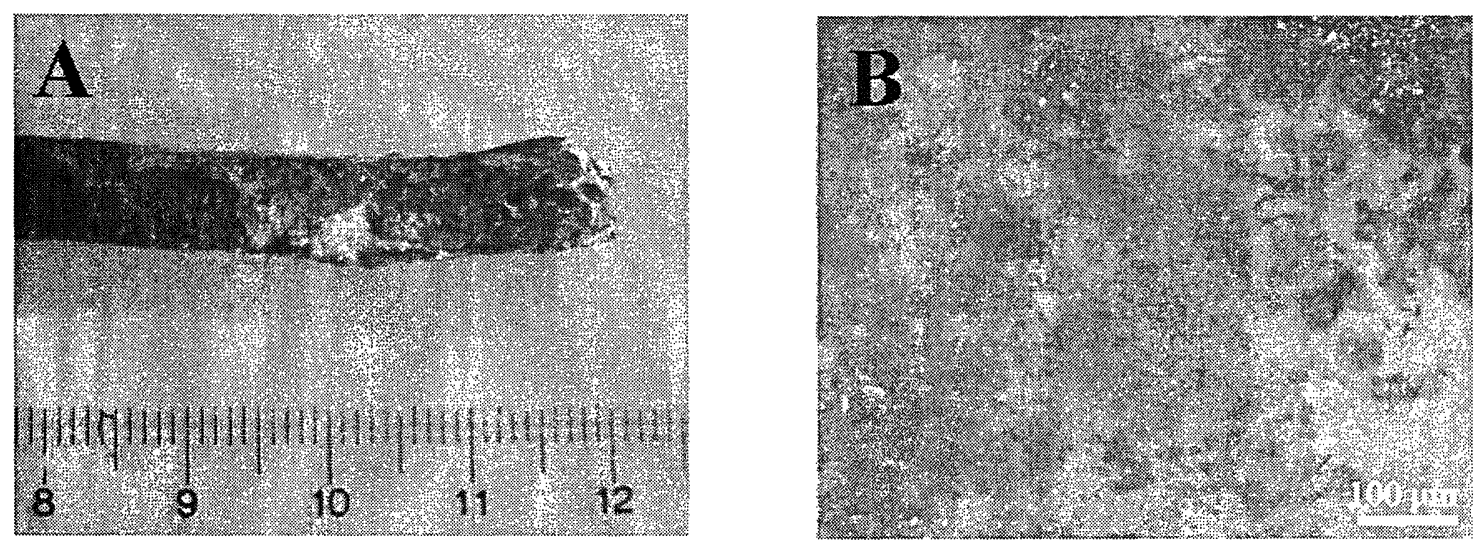

Figura 9.- A) Fotografía de un fragmento de barra de sujeción procedente de una vidriera prerrenacentista (segunda mitad del s. XV). B) Imagen de MO de un fragmento de trabilla de sujeción procedente de una vidriera prerrenacentista (segunda mitad del s. XV).

Figure 9.- A) Photograph of a supporting bar piece from a pre-Renaissance (1 $5^{\text {th }}$ (entury) stained glass window: $B$ ) OM image of a supporting hook piece from a pre-Renaissance (15 century) stained glass window: 
formados como consecuencia de una intensa meteorización. La Figura 10 presenta las diferentes zonas de la barra de sujeción de la Figura 9A en una sección transversal: la zona interior (zona A) corresponde al hierro sin atacar y la más externa (zona B) a la capa de corrosión en la que se detectó un contenido alto de oxígeno y una disminución relativa del porcentaje de hierro, como se pudo comprobar mediante el correspondiente microanálisis EDX (Tabla II). En la Figura $11 \mathrm{sc}$ observa cl aspecto poroso, heterogéneo y estratificado de la costra de corrosión. En dicha costra se detectó por DRX la presencia de las siguientes fases cristalinas: akagancita $(\mathrm{FeO}(\mathrm{OH}))$ y en menor proporción calcita $\left(\mathrm{CaCO}_{3}\right)$ y yeso

$\left(\mathrm{CaSO}_{4} \cdot 2 \mathrm{H}_{2} \mathrm{O}\right)$. Al igual que en las costras de los emplomados, el grado de cristalinidad de las fases cristalinas detectadas es muy bajo, debido a las mismas razones expuestas anteriormente. El hecho de que aparezcan sales de calcio, se puede explicar por el arrastre de dicho clemento desde la masilla (habitualmente se preparaban con calcita) y su posterior depósito en la barra metálica, donde en combinación con el $\mathrm{SO}_{2}$ ambiental podría dar lugar al yeso.

En lo que respecta a la masilla, es probable que su utilización como material de relleno entre los perfiles de plomo y los vidrios coloreados no se realizara de una forma asidua hasta el siglo XVI (13), ya que no se han encontrado referencias que documentaran su uso anteriormente. Sin cmbargo, la práctica del enmasillado es habitual desde entonces y probablemente se llevó a cabo desde las primeras restauraciones de las vidrieras medievales de un modo sistemático. En cualquier caso parece que así tuvo que ser, ya que es frecuente encontrar emplomados más cerrados, de alas más grandes o incluso recubiertos de estaño, parcial o totalmente, en vidrieras anteriores al s. XVI; mientras que en las posteriores a esa fecha es común la ausencia

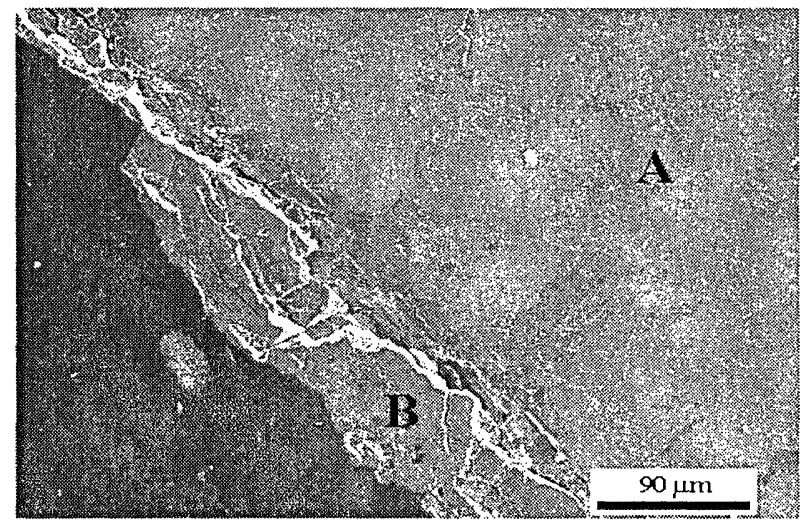

Figura 10.- Imagen de MEB de una sección transversal del fragmento de barra de sujeción de la Figura 9 A

Figure 10.- SEM image of a transiersal section of the supporting bar piece of Figure $9 A$. strong weathering. Figure 10 shows different areas from the supporting bar of Figure $9 A$ in a transversal section: the inner zone (zone A) corresponds to the iron without any attack signs and the outer zone (zone B) the corrosion layer in which a high oxygen content and a relatively diminishing of the iron percentage was detected by EDX microanalysis (Table II). In Figure 11 the porous, heterogeneous and layered corrosion crust can be observed. In that crust, several crystalline phases were detected by XRD: akaganeite $(\mathrm{FeO}(\mathrm{OH})$ ) and low content of calcite $\left(\mathrm{CaCO}_{3}\right)$ and gypsum $\left(\mathrm{CaSO}_{4} \cdot 2 \mathrm{H}_{2} \mathrm{O}\right)$. Similarly to the lead came crusts, the crystallinity degree of the crystalline phases is low, due to the same reasons already explained. The presence of calcium salts can be explained by the dragging of that compound from the puty (calcite was the current raw material) and their further deposition on the metallic bar where, once combined with the environmental $\mathrm{SO}_{2}$, would form gypsum.

In regarding the putty; probably such refilling material between the lead came and the coloured glass pieces was not actually used until the $16^{\text {th }}$ century (13), as could be deduced from the literature: early references about the using of putty have not been found. However, the refilling process with putty is a common task from $16^{\text {th }}$ century and, maybe, it was used as early as the first restorations of the medieval stained glass windows took place. It seems that this is the case even in stained glass window's from centuries before the $16^{\text {th }}$ century; in which closer lead cames, with higher wings or coated by tin (partial or totally) have been found; while in stained glass windows from centuries after the $16^{\text {th }}$ century, the tin coatings lack and more open lead came

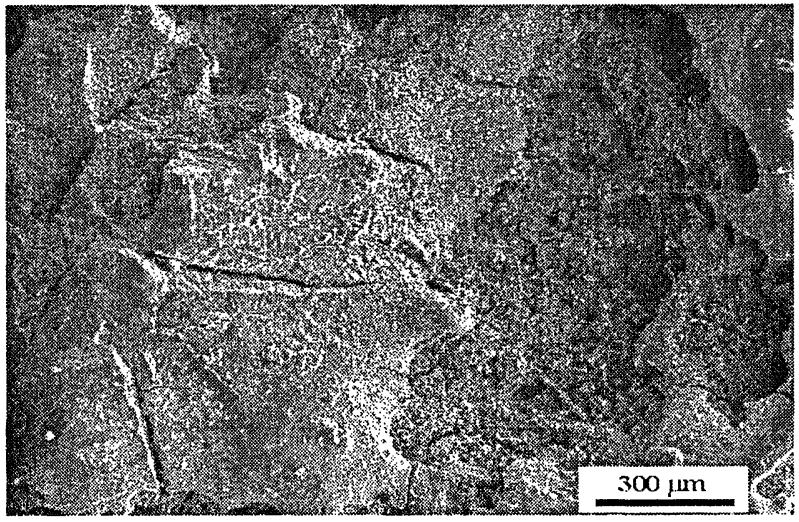

Figura 11.- Imagen de MEB de la costra de corrosión de la trabilla de sujeción de la Figura $9 \mathrm{~B}$.

Figure 11.- SEM image of the corrosion crust of the supporting hook of figure $9 B$. 
del estañado y la utilización de perfiles más abiertos o con alas más pequeñas que permiten el relleno con masilla (10). El uso de la masilla conficre una estabilidad mayor a la vidriera en su conjunto y asegura la fijación de los fragmentos de vidrio coloreado. Cuando no se utilizaba asiduamente, por ejemplo en la arquitectura gótica, el refuerzo de la vidriera y la fijación de los vidrios se confiaban exclusivamente a la red de plomo, al estañado adicional, si se llevaba a cabo, y a los elementos metálicos sustentantes.

En la Figura 12 se muestra la textura de un fragmento de masilla procedente de una vidriera del s. XIX. A pesar de la relativa actualidad de dicha muestra, se detecta una superficie porosa e inhomogénea donde se han desprendido pequeños trozos. El análisis por DRX demostró que la única fase cristalina presente cra la calcita $\left(\mathrm{CaCO}_{3}\right)$ de una pureza muy considerable. Como es sabido, la masilla se prepara habitualmente a partir de ese mineral y una fase orgánica como el accite de linaza o la grasa de pescado. or with smaller wings, which allow the putty refilling, are frequent (10). The use of putty gives more stability to the whole stained glass window and ensures a closer fixation of the coloured glass pieces. When the putty. was not used, for instance in the gothic architecture, the reinforcement of the stained glass window and the fixation of glasses only depended on the lead network, the additional tin coating (if it was applied) and supporting metallic elements.

In Figure 12, the texture of a putty piece from a stained glass window of the $19^{\text {th }}$ century is shown. Despite the relative youth of the sample, a porous and inhomogeneous surface is detected, together with small separated grains. DRX analysis demonstrated that the only crystalline phase present is high purity calcite $\left(\mathrm{CaCO}_{3}\right)$. As is known, the putty is currently prepared from this mineral and an organic phase such as linseed oil or fish fat.

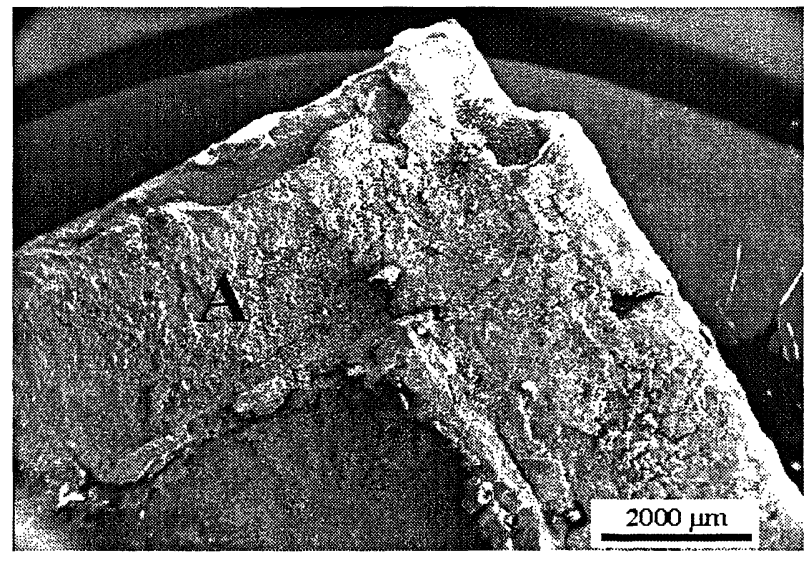

Figura 12.- Imagen de MEB de un fragmento de masilla procedente de una vidriera del s. XIX.

Figure 12.- SEM image of a putty piece from a $19^{\text {th }}$ century stained glass window:

\section{CONCLUSIONES}

Se ha comprobado en diversas muestras de distinta procedencia y cronología que los efectos de la meteorización y en especial de los contaminantes atmosféricos afectan a todos los elementos constituyentes de las vidrieras históricas: vidrios coloreados, red de plomo, barras metálicas de sujeción y masilla de relleno. El proceso de degradación química de los vidrios comienza con una hidratación y avanza según los mecanismos de corrosión conocidos.

Dependiendo del avance del proceso de corrosión, no sicmpre en relación directa con la cronología de la pieza, se forman depósitos de suciedad, hollín, sales insolubles, y la formación de picaduras y costras gruesas. Los clementos metálicos experimentan una corrosión superficial generalizada que se inicia con la

\section{CONCLUSIONS}

Weathering and atmospheric pollution effects on several samples of different provenance and chronology has been proved to be a process affecting all the components of the historical stained glass windows: coloured glasses, lead network, supporting metallic bars and refilling putty: Chemical degradation process of glasses begins with hidratation and then progress through well-know'n corrosion mechanisms. Depending on the advance of the corrosion, which not always is directly related to the piece chronology, deposits of dirty; soot, and insoluble salts are generated, as well as the formation of pits and thick crusts. Metallic elements submit a whole superficial corrosion that begins by forming an oxide layer and then one or several insoluble salt layers, formed by. 
formación de una capa de óxido a la que se superpone una o varias capas de sales insolubles formadas por reacción ente los gases contaminantes y la primera capa de alteración. Los ciclos y choques térmicos originan tensiones mecánicas que pueden evolucionar a la formación de microgrietas que fragilizan los metales y favorecen su corrosión. La masilla se contrae y finalmente se desprende de su posición, dejando libres los huecos entre los fragmentos de vidrio y el emplomado. La porosidad que presentan y el contacto con los agentes de meteorización favorecen su degradación química y desmoronamiento.

Se ha puesto de manifiesto la importancia del impacto ambiental en la conservación integral de las vidrieras históricas y la necesidad de abordar su preservación teniendo en cuenta todos los elementos que las constituyen, ya que en los procesos de corrosión de cada material intervienen, al menos parcialmente, los productos de degradación de los otros, que ejercen un efecto sinérgico desfavorable.

\section{AGRADECIMIENTOS}

Los autores agradecen el apoyo de la Red Temática de Patrimonio Histórico y Cultural del CSIC. M.G.H. agradece al CSIC-FSE la financiación de un contrato postdoctoral I3P. reaction between gaseous pollutants and the first alteration layer. Cycles and thermal shocks give rise to mechanical stresses that could form microcracks favouring fragility and corrosion of the metals. The putty shrinkages and gives off from their original position, leaving free space between the glass pieces and the lead cames. The porosity and continuous contact of the putty with weathering agents favour their chemical degradation and wearing away.

The importance of the environmental impact on the integral conservation of the historical stained glass windows has been pointed out. Moreover, we have presented the need to face their preservation along the time taking care of all the elements that forms part of the stained glass windows. The reason is related to the corrosion process of each material, in which the degradation products of the other develops, at least partially, a negative synergic effect.

\section{ACKOWLEDGEMENTS}

The authors gratefully acknowledge support provided by the Historical and Cultural Heritage Thematic Network from CSIC. M.G.H. also acknowledges financing support from CSIC-ESF through an I3P postdoctoral contract.

\section{BIBLIOGRAFÍA}

(1) M. Pérez y Jorba, J.P. Dallas, C. Bauer: Deterioration of stained glass by atmospheric corrosion and microorganisms. J. Mater. Sci. 15 (1980), pp. 1640-1647.

(2) G.A. Cox, A.M. Pollard: The multivariate analysis of data relating to the durability of medieval window glass. Revue d'Archéometrie 5 (1981), pp. 119-128.

(3) K.J.S. Gillies, G.A. Cox: Decay' of medieval stained glass. Part. 2. Relationship between the composition of the glass, its durability' and the weathering products. Glastech. Ber. 61 (1988), pp. 101-107.

(4) M. Schreiner: Deterioration of stained medieval glass by atmospheric attack: Pt. 1. Scanning electron microscopic investigations of the weathering phenomena. Glastech. Ber. 61 (1988), pp. 197-204.

(5) M. Schreiner: Deterioration of stained medieval glass by' atmospheric attack: Pt. 2. Secondary ion mass spectrometry analy sis of the naturally weathered glass surfaces. Glastech. Ber. 61 (1988), pp. 223-230.

(6) W. Müller: Corrosion phenomena of medieval stained glasses. Bol. Soc. Esp. Ceram. Vidr. 31-C, Vol. 1 (1992), pp. $219-239$.

(7) N. Carmona. Estudio de los procesos de alteración de vidrieras históricas y' de los tratamientos para su restauración y protección. Tesis doctoral, Valladolid, julio 2002.

(8) J.M. Fernández Navarro: "Causas del deterioro físico y químico de los vidrios históricos". Jomadas Nacionales sobre Restauración y Conservación de Vidrios. J.M. Fernández Navarro y P. Pastor Rey de Viñas (Eds.), pp. 17-37, La Granja de San Ildefonso, 2000.

(9) J.M. Fernández Navarro: Procesos de alteración de las vidrieras medievales. Estudio y' tratamientos de protección. Mater. Construcc. Vol. 46 (1996), pp. 5-25.

(10) F. Cortés: Estudio del plomo medieval en las vidrieras del monasterio de Pedralbes (Barcelona). Mater. Construcc. Vol. 50 (2000), pp. 85-95.

(11) V.B. Rambush: The lead cames of stained glass windows: purpose, problems and preservation procedures. Technology and Conservation Vol. 8, n 3 (1993), pp. 46-49.

(12) J.G. Castaño González, C. López de Azcona, M. Morcillo Linares: Deterioration of ancient metallic elements taken from Toledo cathedral. Rev. Metalurgia 37 (2001), pp. 519-527.

(13) J.T. Smith. Antiquities of Westminster. Londres, 1807. 